\title{
Ser, estar y haber en el aula de español como lengua extranjera
}

Martha

J urado Salinas

CEPE-UNAM

INTRODUCCIÓN

Los verbos haber, ser y estar comparten tres rasgos que los diferencian del resto de los verbos del español:

1. No nombran ninguna acción o proceso.

2. Su significado léxico es muy poco concreto y se refiere a la mera existencia de una entidad o bien a sus características.

3. Tienen funciones auxiliares.

Como afirma Molina Redondo, "es ya tópica la afirmación de que uno de los puntos más complicados de la gramática del español es el constituido por los usos de los verbos ser y estar." ${ }^{1}$ La complejidad proviene de varios factores: en primer término, la existencia en español de dos verbos para funciones y contenidos que en otras lenguas cumple y transmite un solo verbo; en segundo lugar, la diversidad de enfoques y explicaciones que tratan de dar cuenta de la complejidad del fenómeno, unas veces apelando a un criterio único que resulta insuficiente y otras convirtiéndose en enumeraciones exhaustivas de ejemplos, excepciones, situaciones posibles, vacilaciones, etc.; y en tercer lugar, la ausencia de una explicación clara y -sin lista anexa de excepciones-aplicada al diseño de materiales para la enseñanza del español a extranjeros.

Indudablemente, resulta difícil buscar el equilibrio entre la simplicidad y la claridad de una propuesta teórica y el respeto debido a los fenómenos lingüísticos que se pretenden explicar. En este documento se postula la hipótesis de que la complejidad de los fenómenos lingüísticos requieren de una comprensión profunda, por parte del

J. A. de Molina Redondo y J. Ortega Olivares, Usos de ser $y$ estar, p. 9. 
2 Si queremos comprender el mundo debemos expresar las cosas en términos que podamos entender. Reducir algo es como traducirlo a un lenguaje más inteligible. Una vez aplicada la reduoción, los fenómenos resultan más manejables y menos misteriosos. (...) Las explicaciones más sencillas se consideran «inferiores» porque están más cerca de los hechos básicos que conocemos. Pero también podríamos tildarlas de «superiores» porque se elevan por encima de los detalles irrelevantes y confusos para llegar a la verdad esencial de las cosas. (Nicholas Fearn, Zenón y la tortuga, p. 19) .

3 Del latín Habere "tener, poseer". Sustantivado: haber "bienes", Corominas, Breve diccionario etimológico de la lengua castellana, p. 1220-50.

4 La relación entre el sentido locativo y el existencial fue apuntado desde hace décadas por semantistas como Lyons, quien sostiene que las construcciones posesivas, locativas y existenciales están íntimamente vinculadas porque tiene un origen locativo común: In many and perhaps in all, languages existential and possessive constructions derive from locatives. It rests upon the assumption that the copula is a purely grammatical element which carries distinctions of tense, mood and aspect in the surface structure of certain classes of stative sentences. John Lyons, "A note on possesive, existential and locative sentences", en Foundations of Language, p. 390. profesor y de los diseñadores de materiales, de conceptos lingüísticos que a veces poseen un al to grado de abstracción, pero también un amplio poder de generalización. Se propone que, adoptando una perspectiva que conjuga sintaxis, semántica, conocimiento del mundo y cognición general, se puede al canzar este nivel explicativo. Así mismo se recurre al uso de metáforas físicas como mecanismo cognitivo que se utiliza para procesar información abstracta a partir de conceptos más concretos, simples y familiares.

Esta comprensión proporcionaría la base para que profesores y diseñadores realizaran su tarea práctica, aterrizándola en explicaciones accesibles complementadas con ejercicios que propiciaran la adquisición del uso lingüístico contextualizado. Este sería, al final de cuentas, el reto de una gramática pedagógica: reducir la complejidad de las explicaciones lingüísticas, sin caer en reduccionismos simplistas. ${ }^{2}$

En este documento se desarrolla primero una explicación contrastada entre los usos de haber/ estar, después entre ser/ estar, para ejemplificar cómo esta concepción, aparentemente compleja, podría aplicarse al trabajo en el aula de español como lengua extranjera.

\section{CONTRASTE HABER / ESTAR}

La forma impersonal hay procede de la adición del adverbio anticuado hi (escrito muchas veces $y$ ) a la forma de tercera persona del verbo posesivo latino habere, ${ }^{3}$ es decir, a la forma ha («iiene»). Se decía en español antiguo hi ha o ha hi > ha $+y>$ hay, ( ha ahí = tiene ahí). Existen expresiones similares en otras lenguas: vi (ci) ha / c'e/ ci sono (italiano), il y a(francés), est gibt (alemán), there is / are (inglés) ${ }^{4}$

El verbo estar proviene del latín stare sponer o estar de pie». De expresar una permanencia pasajera de un sujeto en un lugar, pasó a designar el estado transitorio en el que se halla dicho sujeto.

Observemos el cuadro: 
Cuadro 1

Usos de haber y estar

\begin{tabular}{|l|l|}
\hline \multicolumn{1}{|c|}{$\begin{array}{c}\text { Haber } \\
\text { (I mpersonal })\end{array}$} & \multicolumn{1}{|c|}{ Estar } \\
\hline \multicolumn{1}{|c|}{ Existencia } & \multicolumn{1}{|c|}{ Local ización } \\
\hline 1a. Hay oro & 1b.* Está oro \\
2a. hay tristeza & 2b.* está tristeza \\
3a. hay una casa & 3b. ? está una casa [ahí] \\
4a. hay tres casas & 4b. ? están tres casas [ahí] \\
5a. hay al gunos perros & 5b. ? están al gunos perros [ahí] \\
6a. hay muchas cajas & 6b. ? están muchas cajas \\
7a. hay cajas & 7b.* están cajas \\
8a.*5 hay el puente & 8b. está el puente [ahí] \\
9a.* hay mi hijo & 9b. está mi hijo [ahí] \\
10a.* hay esta casa & 10b. está esta casa [aquí] \\
11a. hay la posibilidad de que... & 11b. está la posibilidad de que.. \\
\hline
\end{tabular}

El verbo haber en su uso impersonal introduce un tema en el discurso, presenta una entidad genérica, comunica la existencia de al go. Se combina con la forma singular o plural de un sustantivo sin artículo 1a., 2a., 7a. También se puede combinar con un sustantivo introducido por artículo indefinido para hacer referencia a un miembro de una clase de entidades $3 a$.

Lógicamente, no se suel e combinar con el artículo definido, porquesi sabemosidentificar el objeto lo suficiente para marcarlo con el artículo definido, lo damos por conocido (segunda mención), 8a.

Como puede observarse en los ejemplos, hay y estar se complementan en varios aspectos:
El asterisco, indica que es una secuencia agramatical para un hablante nativo. 
Cuadro 2

Estructura sintáctica: Hay + Objeto Directo (O. D.)
Estructura sintáctica: Sujeto + estar $+[\ldots .$.

\begin{tabular}{l} 
Valor semántico existencial. Verbo neutro de \\
estado equi valente a ser o existir. \\
\hline Compatible con O.D. no contables. [1a., 2a.] \\
\hline $\begin{array}{l}\text { Compatible con O.D. con determinante inde- } \\
\text { finido. [3a., 4a., 5a., 6a.] }\end{array}$ \\
$\begin{array}{l}\text { Compatible con O.D. plural sin determinante. } \\
\text { [7a.] }\end{array}$ \\
\hline $\begin{array}{l}\text { Incompatible con O.D. sustantivos con deter- } \\
\text { minante definido (artículos determinados, } \\
\text { posesivosy demostrativos) [8a., 9a., 10a.] }\end{array}$
\end{tabular}

Valor semántico de locación o situación.

Incompatible con sujetos no contables. [1b., 2b.]

Incompatible con sustantivos con determinanteindefinido. [3b., 4b., 5b., 6b.]

Incompatible con sustantivos plurales sin determinante. [7b.]

Compatible con sustantivos con determinante definido (artículos determinados, posesivos y demostrativos). [8b., 9b., 10b.]

Los ejemplos 11a. y 11b. nos muestran que el sintagma nominal (SN) que complementa a hay puede llevar artículo determinado cuando se introduce una entidad conocida en un contexto nuevo. Ello requiere la mayor elaboración del SN, ya sea mediante modificadores o complementos prepositivos u oracionales:

(12) *Hubo la crisis.

(13) Hubo la crisis que todos esperaban.

(14) Hay la necesidad de actual izar a los profesores.

(15) — ¿Qué hay de comer?

- Hay fruta y hay un pedazo de pizza.

-Es que se me antoja al go caliente.

-Ah... hay la sopa que sobró ayer, te la puedes calentar.

LoS SN que complementan a hay presentan peculiaridades que han motivado vacilación respecto a su estatus gramatical.

Los argumentos a favor de su estatus de O. D. son:

- Sesustituyen por las formas objetivas directas /o/la/ los/ las (lo hay, las hay, etcétera). 
- Aparece pospuesto al verbo, es decir, sigue el orden de palabras «normal »en castellano para el grupo Verbo + O. D.

Los argumentos a favor de su estatus de sujeto son:

- Se trata de un verbo de estado que denota existencia. Los verbos de estado son intransitivos, no tienen objeto directo.

- En muchas zonas, es frecuente (incluso en niveles cultos) hacer concordar en número y persona lossintagmas nominales pospuestos (o antepuestos en oraciones interrogativas) con el verbo; es decir, en estas zonas, el objeto directo parece sentirse como un sujeto:

(16) * Hubieron cinco marchas en la ciudad.

Son usos proscritos académicamente, pero ya registrados en lengua escrita.

También es incorrecta la forma concordada en primera persona del plural:

(17) * ¿Cuántos maestros habemos en el Centro?

- Las construcciones con hay rechazan la transformación en voz pasiva. Si su estructura sintáctica fuera «verbo transitivo + O.D.», sería transformable en «sujeto + ser + participio».

(18) * Muchos niños son habidos en el patio.

- Los objetos directos animados que complementan a hay carecen de preposición a. Esterasgo se relaciona con la indeterminación característica de los sintagmas nominales que lo complementan.

(19) * Hay a una persona esperando.

La caracterización etimológica, sumada al análisis de los usos complementarios con estar en los niveles semántico y sintáctico, y a las tendencias anómal as de su uso en el español hablado actual, nos llevan a plantear la hipótesis de que la función semántica de haber es introducir elementos en el discurso - generalmente entidades de primer orden que ocupan un lugar en el espacio ${ }^{6}-$ desde una perspectiva que "enfoca"la real idad externa.
Un nombre prototípico denota un objeto localizable en el espacio (visible, tangible, concreto, delimitado, etc.) y estable en el tiempo. Según Lyons, los objetos son evaluados típicamente en términos de su existencia y son denominados entidades de primer orden.

Un verbo denota situaciones, estados de cosas, eventos, procesos, circunstancias, etc., que se desarrollan en el tiempo y en el espacio y de las cuales se dice que tienen lugar.Lyons las llama entidades de segundo orden. Las situaciones poseen desarrollo cronológico, por ello se dice que los verbos que las denotan tienen un "perfil temporal". 
Dicha perspectiva "impersonal izada" se ubica en un punto identificado con un hablante virtual que "tiene aquí" (como en el sentido etimológico original) su punto de vista desde el cual enfoca la realidad que lo rodea; sin embargo, la perspectiva no corresponde al "yo" gramatical (ni a ninguna otra persona), razón por la cual las secuencias con los determinantes posesivos y demostrativos -éstos últimos deíciticos respecto a la primera persona - resultan agramatical es. La perspectiva corresponde al "yo hablante", pero no corresponde al "yo gramatical". Esta contradicción inherente hace que la tendencia sincrónica sea hacia la identificación de ambas entidades en una sola que conjugue la perspectiva del hablante con la primera persona, de ahí el uso anómalo de habemos y las vacilaciones en el estatus de objeto o sujeto del sintagma que le sucede; es decir, al no existir un sujeto gramatical que enfoque la realidad, el hablante tiende a colocar en esa posición al único SN que aparece en la secuencia.

Gráficamente, esta hipótesis de la fal ta de coincidencia entre "la perspectiva impersonal izada" y la "persona gramatical" podría representarse en una imagen de esta manera:
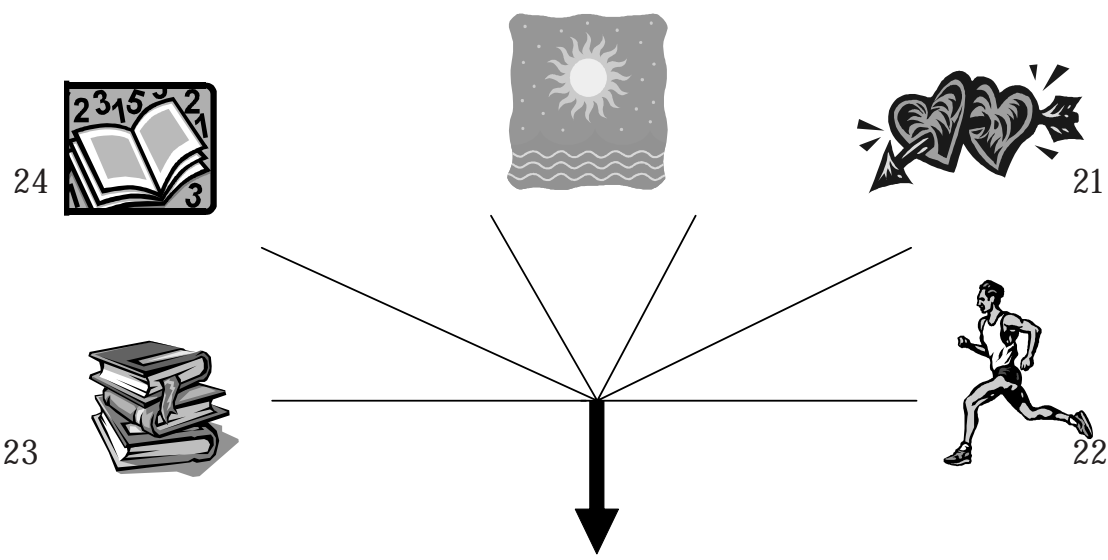

23

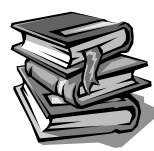

HAY. Punto de perspectiva (Diferente persona gramátical).

Fig. 1. 
(20) Hay sol, hay mar, hay agua (sustantivos no contables concretos).

(21) Hay amor (sustantivo no contable abstracto).

(22) Hay un corredor (sustantivo contable).

(23) Hay libros (sustantivo contable plural).

(24) Hay un libro (sustantivo contable singular).

Como puede observarse, la combinación semántica es amplia. Sus restricciones combinatorias se relacionan con las personas gramaticales (*hay mi hijo, *hay su opinión, *hay esta casa, *yo hay, *hay ellos, ?nosotros habemos) y con la incompatibilidad con la función característica del artículo definido como introductor en el discurso de entidades ya mencionadas (*hay la casa).

Es decir, la perspectiva impersonal izada hace visible a la interpretación semántica una realidad "congelada" en el tiempo (semejante a una fotografía), desvinculada de cualquier persona gramatical o al gún otro elemento deíctico o anafórico. ${ }^{7}$

A reserva de ampliar en la siguiente sección, adelantaremos que estar del imita o circunscribe un espacio y tal delimitación trae como consecuencia una ubicación espacial o temporal; requiere, por tanto, de un argumento locativo. Se predica de las entidades enfocando el espacio que ocupan, es por ell o que la construcción sintáctica corresponde al esquema sujeto + verbo + estructura gramatical o sintagmática correspondiente a un argumento locativo.

(25) Mi casa está ahí.

(26) Mi casa está en la colonia Anzures.

\section{CONTRASTE SER - ESTAR}

En la tradición gramatical, se hace distinción entre los usos «predicativo»y «atributivo» de los verbos copulativos. El verbo ser funciona predicativamente cuando significa «haber»o «existir».
a. Ser o no ser
(Existir o no existir)
b. Dioses el quees (Dios es el que existe)

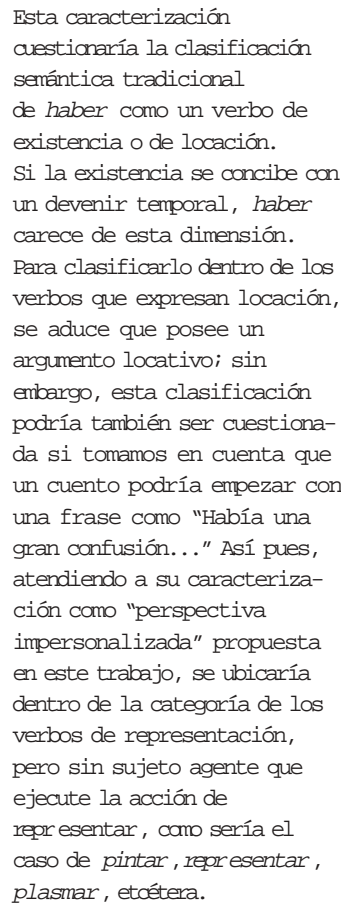


8 Dentro de la gramática cognoscitiva, un dominio es un ámbito coherente de conocimiento que funciona como marco para conceptos más específicos. Sitúa el significado en un entorno conceptual. Se trata de una especie de "telón de fondo" sobre el que se proyecta el concepto en cuestión.

Por ejemplo, el sustantivo martes se enmarca dentro del dominio de los días de la semana, rooillta

dentro del dominio de partes del cuerpo, o de las articulaciones, etcétera. (J. M. Cuenca y Joseph Hilfety, Introcucción a la lingüistica cognitiva, p. 72.)

Las formas del verbo ser fueron producto de la fusión de las de dos verbos latinos. La mayor parte procede del latín esse, pero las demás, incluyendo el futuro, el pospretérito, los presentes de subjuntivo e imperativo, vienen del latín sedere "estar sentado" que debilitó en castellano y portugués su sentido hasta convertirse en sinónimo de estar y luego ser.

Seinterpreta también como predicativo la expresión de la hora ( son las cuatro) o la acepción en que significa «ocurrir», <real izarse»( /a conferencia es en el auditorio).

En el uso atributivo, un verbo copulativo atribuye a un sujeto la cualidad o circunstancia expresadas por un adjetivo o un nombre (el niño estáenfermo, J uan esmecánico).

En su uso predicativo, estar es caracterizado como un verbo intransitivo, con la significación general (derivada de su origen en el verbo latino stare «estar en pie») de 〈permanencia», «situación»o 〈posición local»:

(28) J uan está con su hermano.

Estar atributivo es definido como un verbo vacío, copulativo, que permite vincular a los sujetos con ciertos predicados concebidos como 'estados', o propiedades transitorias, cambiantes o circunstanciales.

(29) J uan está cansado.

Desde una perspectiva semántica, la diferencia predicativa y atributiva podría superarse caracterizando a estar como una semicópula que ubica una entidad en un dominio ${ }^{8}$ físico (p. ej. 25 y 26) o abstracto (si consideramos a "su hermano" y al cansancio como un locus en el cual se inserta a J uan).

Por otra parte, el verbo ser ${ }^{9}$ puede aparecer en los siguientes contextos:

\section{Cuadro 3}

\begin{tabular}{|c|c|c|}
\hline Ejemplo & $\begin{array}{c}\text { Categoría } \\
\text { postverbal }\end{array}$ & $\begin{array}{c}\text { Relaciones } \\
\text { semánticas }\end{array}$ \\
\hline \begin{tabular}{c|c|} 
(30) a. Ese hombre es J uan. \\
b. J uan es el médico de la \\
escuela. \\
J uan es él (él es J uan).
\end{tabular} & SN & $\begin{array}{c}\text { Identificación } \\
\text { ¿Quién es? }\end{array}$ \\
\hline c. J uan es un doctor. & SN & $\begin{array}{c}\text { Identificación } \\
\text { ¿Quién es? }\end{array}$ \\
\hline d. J uan es doctor. & SN & $\begin{array}{c}\text { Membresía a } \\
\text { una clase }\end{array}$ \\
\hline e. J uan es comunista. & SN & $\begin{array}{c}\text { Profesión } \\
\text { ¿Qué es? }\end{array}$ \\
\hline
\end{tabular}


Cuadro 3

(continuación)

\begin{tabular}{|c|c|c|}
\hline Ejemplo & $\begin{array}{c}\text { Categoría } \\
\text { postverbal }\end{array}$ & $\begin{array}{c}\text { Relaciones } \\
\text { semánticas }\end{array}$ \\
\hline f. J uan es inteligente. & SA & $\begin{array}{c}\text { Asignación de una } \\
\text { propiedad }\end{array}$ \\
\hline $\begin{array}{c}\text { g. El coche es de J uan. } \\
\text { El coche es mío. }\end{array}$ & $\begin{array}{c}\text { SP } \\
\text { pronombre }\end{array}$ & $\begin{array}{c}\text { ¿Qué es? } \\
\text { Posesión }\end{array}$ \\
\hline h. La reunión será a las 12. & SP & temporalidad \\
\hline $\begin{array}{c}\text { i. La reunión es en el salón } \\
\text { doce. } \\
\text { La reunión es ahí. }\end{array}$ & $\begin{array}{c}\text { SP } \\
\text { adverbio }\end{array}$ & locación \\
\hline $\begin{array}{c}\text { j. La casa fue construida } \\
\text { por sus antepasados. }\end{array}$ & $\begin{array}{c}\text { perífrasis } \\
\text { (pasiva) }\end{array}$ & pasividad \\
\hline
\end{tabular}

El verbo copulativo estar, por su parte, aparece en contextos como:

\section{Cuadro 4}

\begin{tabular}{|c|c|c|}
\hline Ejemplo & $\begin{array}{c}\text { Categoría } \\
\text { postverbal }\end{array}$ & $\begin{array}{c}\text { Relaciones } \\
\text { semánticas }\end{array}$ \\
\hline (31) a. J uan está loco. & SA & $\begin{array}{c}\text { Propiedad transitoria. } \\
\text { Comparación deun estado } \\
\text { del sujeto con otro estado. }\end{array}$ \\
\hline $\begin{array}{c}\text { b. J uan está en el salón. } \\
\text { La casa está ahí. }\end{array}$ & $\begin{array}{c}\text { SP } \\
\text { Adverbio }\end{array}$ & $\begin{array}{c}\text { Ubicación espacial, } \\
\text { localización. }\end{array}$ \\
\hline $\begin{array}{c}\text { c. La casa está construi- } \\
\text { da sobre basalto. }\end{array}$ & $\begin{array}{c}\text { Perífrasis (pasiva } \\
\text { de recultado) }\end{array}$ & Estado alcanzado. \\
\hline d. Juan está comiendo. & Perífrasis & Progresión. \\
\hline
\end{tabular}

Como puede observarse, sólo ser puede combinarse con sustantivos en la posición posverbal 30a, b, c, d; por tanto, esta combinación no presenta problemas para la enseñanza del español.

Las estructuras de ser y estar coinciden en $30 \mathrm{~h}=31 \mathrm{a}$ (verbo copulativo + adjetivo), 30j = 31c (voz pasiva) y, cuando el sujeto denota un evento, también coinciden las estructuras 30i $=31$ b (verbo copulativo + advervios o complemento de lugar). De estas tres estructuras coincidentes, la que causa problema al ser trabajada con los 
10 Los criterios más comunes que se han utilizado para explicar la diferencia entre ser y estar son: esencia/estado; esencia/accidente: clasificación/descripción; intrínseco/extrínseco; abjetivo/subjetivo; habitual/ocasional; permanente/cambiante.

11 El aspecto perfecto se manifiesta en el significado de los verbos (aspecto léxico) o en la flexión verbal (aspecto gramatical) e indica acciones acabadas o más bien, los estados resultantes de situaciones previas:

"Este hecho constituye, sin cuda, la característica más notable del Perfecto: la focalización de un momento posterior al de la situación proporcionada por el verbo en en forma no finita.

De esta característica del Perfecto, se deriva otra: cuando el tipo de predicado lo permite, un Perfecto puede ser parafraseado por las perífrasis resultativas tener + participio y estar + participio...," (Luis García Fernández, El aspecto gramatical en la conjugación, p. 52.)

12 Ronald Langacker, Foundations of Cognitive Grammar: Theoretical Prerequisites. alumnos extranjeros es la primera (verbo copulativo + adjetivo).

La diferencia en los usos de ser/estar + adjetivo se plantea general mente en términos de temporalidad: ${ }^{10}$ con ser, la propiedad atribuida es permanente (30f), mientras que con estar, es presentada como temporalmente relevante (31a).

En este documento se propone la hipótesis de que ser es un verbo copulativo prototípico, semánticamente vacío (esquemático), mientras que estar, como ya se mencionó, es un verbo «semicopulativo». Las semicópulas cumplen la misma función que las cópulas, pero a diferencia de éstas, añaden a las construcciones en las que aparecen un modicum de significado, en el caso de estar, de tipo aspectual perfecto. ${ }^{11}$

\section{CARACTERIZACIÓN ASPECTUAL DE LOSVERBOS COPULATIVOS}

Desde la perspectiva cognoscitiva, ${ }^{12}$ ser es caracterizado como un tipo de verbo que designa un proceso al tamente esquemático, es decir, que tiene poco contenido semántico más allá del cual se le caracteriza como clase.

Este val or semántico especial lo habilita para desempeñar roles gramatical es particulares, uno de ellos, Ilenar el perfil temporal requerido por una oración finita cuyo contenido más importante es suministrado por una predicación atemporal (sustantivo, adjetivo o participio). Como predicación que denota un proceso que se desarroIla en el tiempo, sirve como núcleo oracional, pero se trata sólo de un proceso "esquelético" cuyos estados componentes no están identificados. El adjetivo (u otra predicación atemporal), Ilena el esqueleto, es decir, designa la secuencia de configuraciones de cada etapa del proceso especificadas en detalle.

Cuando se combinan con ser, estas predicaciones sustantivas, adjetivas o participiales se igualan con los estados componentes del verbo copulativo. La expresión compuesta resultante (p. ej. es médico, es alto, es explicado, etc.) hereda el perfil temporal del verbo y designa un proceso específico. De esta manera, ser convierte la 
predicación atemporal denotada por el sustantivo, y la estativa denotada por adjetivo y por el participio, en un proceso imperfecto o durativo.

Así pues, los verbos copulativos serían el recurso lingüístico para dotar de tiempo y persona a una categoría que carece de ellos (adjetivo, sustantivo o participio). Un adjetivo denota una cualidad (p. ej. grande) que, como tal, es susceptible de cambiar; un participio denota un estado al canzado (p. ej. embellecido); un sustantivo denota prototípicamente un objeto ubicable en el espacio, estable en el tiempo y por tanto carente de desarrollo temporal. Adjetivos y partici pios se combinan tanto con ser como con estar. Por su carácter estable en el tiempo, los sustantivos sólo se combinan con ser, que denota un proceso al tamente esquemático que también se extiende imperfectivamente (durativamente). Así, en el ejemplo J uan es el médico de la escuela, el SN [el médico de la escuela] adquiere, a través de la cópula, tercera persona (singular), duración y ubicación temporal.

Recurriendo a una metáfora, el carácter esquelético del verbo ser podría representarse como un ferrocarril vacío que se desplaza en el tiempo. La materia contenida en los vagones representaría el contenido semántico del objeto o la cualidad que, al "montarse" en el tren, adquiere un perfil temporal. ${ }^{13}$

(32) J uan es alegre.

(33) J uan es médico.
13 Si se concibe la existencia-como ya se mencionó anteriormente como un devenir temporal, el verbo ser podría ser ubicado cabalmente dentro de este campo; mientras que estar se ubicaría dentro de los verbos que denotan locación.

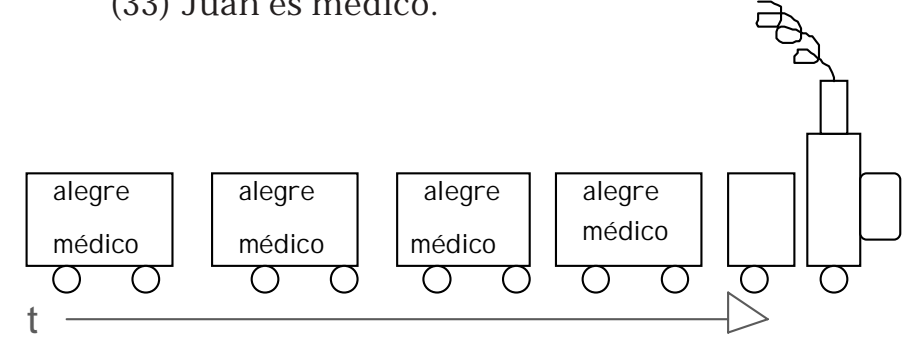

(tiempo)

Fig. 2.

El verbo estar (en todossus usos, excepto en el de perífrasis progresiva) tiene un valor semántico de locación. Cuando se combina con adjetivos, denota una situación o estado que se al canza gracias a un proceso reconocible o no y que es especificado por la expresión postverbal 
14 I. Bosque, "Sobre el aspecto en los adjetivos y en los participios", en Tiempo y aspecto en español, p. 33.

15 No importa la ubicación temporal deíctica de la predicación estativa (estuvo, estaremos, estoy), siempre se sobreentiende que un intervalo temporal es enfocado por la percepción de un conceptualizador personalizado (a diferencia de la "perspectiva impersonalizada" que se produce con el verbo haber). Se trata, en este sentido, de lo que se conoce tipológicamente como «resultativas que describen estados visualmente perceptibles». como situación física o como estado psíquico, fisiológico, etcétera (está sano/triste/ de buen humor/con gripe/ bien, etcétera).

La lengua española marca a través del pronombre se cambios de estado, es por ello que a cada verbo pronominal corresponde una construcción perifrástica con estar que denota el estado resultante del cambio:

(34) a. se casó $\rightarrow$ está casado

b. se enojó $\rightarrow$ está enojado

c. se murió $\rightarrow$ está muerto

Los conceptos de "estado"y "situación" comparten un originario sentido físico, locativo, como apunta Bosque, ${ }^{14}$ respecto al hecho de que la propagación de estar en la historia del español se produjo como extensión de los usos locativos. Bosque considera que la perfectividad se ha desarrollado muy probablemente en la lengua como metáfora física de la locación.

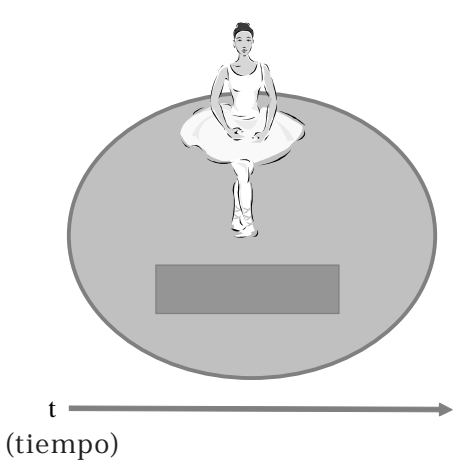

Recurriendo nuevamentea una metáfora, podríamos representar el significado de "estado" como una circunscripción en el espacio o en el tiempo. El significado etimológico de estar «estar de pie»nos remite a una ubicación central dentro de ese dominio cognoscitivo.
Fig. 3.

Cuando se combina con estar, la atemporalidad de la cual idad denotada por el adjetivo es del imitada por el verbo. Se produce un efecto perfectivizador, delimitador, de estados resultantes que, una vez circunscritos, son relacionados con la percepción directa de un conceptualizador real o virtual cuya perspectiva se encuentra ubicada en al gún punto del ejetemporal (presente, pasado ofuturo). ${ }^{15}$

Uno de los requisitos para usar un adjetivo con el verbo estar es que la propiedad o cual idad sea evidente a la 
percepción de los sentidos y que pueda ser concebida como un estado susceptible de ser al canzado como producto de un cambio:

(35) a. estar al egre

b.* estar inteligente

Es decir, sólo las cual idades visibles que no se extienden indefinidamente en el tiempo, sino que ingresan a la circunscripción estativa por al gún cambio de estado, son susceptibles de combinarse con estar. ${ }^{16}$

Una caracterización aspectual de este tipo daría también cuenta de por qué estar no puede entrar en ciertas combinaciones. La perfectividad y la delimitación resultan semánticamente incompati bles con la "masa" denotada por los sustantivos continuos o no contables (oro, tristeza) y con la "replicabilidad" con la que crecen los sustantivos plurales, cuantificados o con determinante indefinido (cajas, tres casas, algunos perros). ${ }^{17}$

Metafóricamente, sery estar podrían representarse de la siguiente manera: el estado imperfecto denotado por ser cambia y da lugar a un estado. El momento del cambio se gramaticaliza mediante un verbo pronominal.
16 En la gramática cognoscitiva se considera que la gramática es un vehículo de la

semántica, y como tal, no sólo el léxico, sino también la sintaxis, reflejan el conocimiento que el

conceptualizador tiene del mundo. En el caso de los usos con estar, el conocimiento enciclopédico determina que hay estados susceptibles de cambiar; o estados que pueden conceptualizarse como permanentes 0 cambiantes (p. ej. es casadoy está casado).

17 Cfr. cuadro 1, p. 75.

Fig. 4.

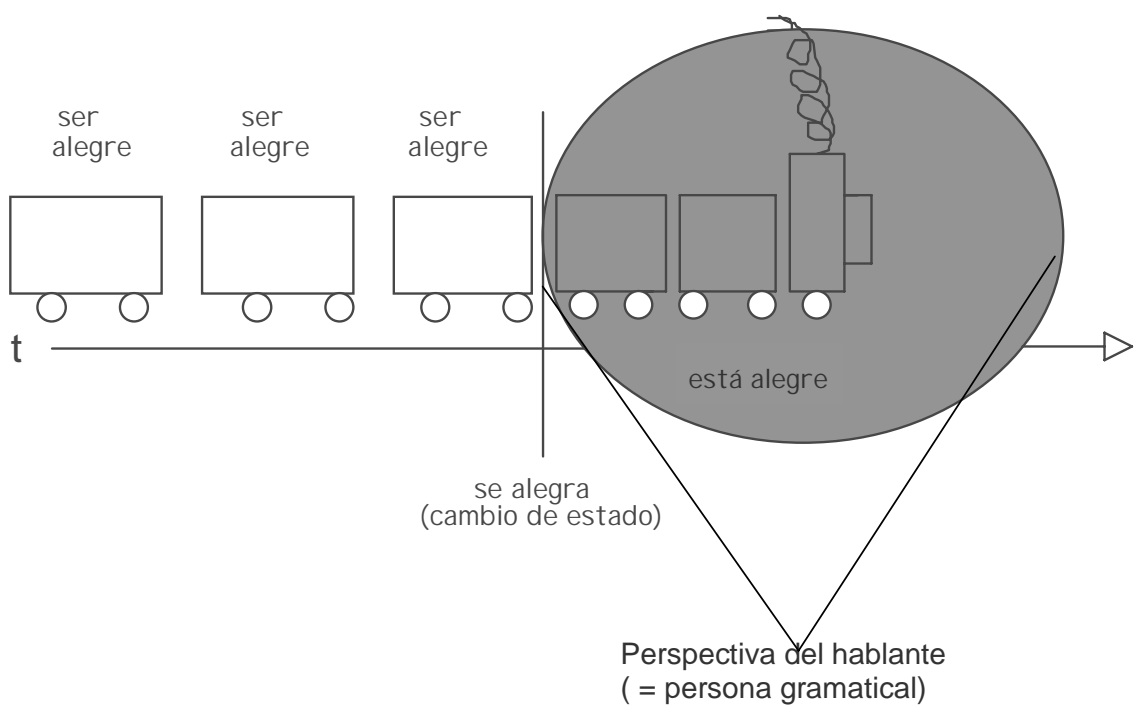


PROPUESTA PARA TRABAJ AR LOS VERBOS HABER, ESTAR Y SER EN EL AULA DE ESPAÑOL LENGUA EXTRANJ ERA

Por motivos de espacio, no desarrollaré una unidad didáctica completa sobre el tema; sin embargo; señalaréal gunos puntosque -considero- deben tomarseen cuenta, alaluz de las hipótesis planteadas en el presente documento:

1. Cada uno de los verbos debe ser presentado individualmente, en unidades distintas con el fin de identificar su conjugación y sus usos característicos.

2. El orden de presentación sería: haber, estar, ser.

3. Haber se introducirá mediante la pregunta ¿qué hay?, haciendo que al guien se asuma como una cámara fotográfica que fuera colocada al centro del salón (no una persona gramatical) y enfocara qué se ve a través del lente.

4. Estarse introducirá con la pregunta ¿dónde está? La perspectiva se traslada a una entidad que sintácticamente pasa a funcionar como sujeto gramatical y que se ubica en relación con el espacio externo.

5. Ser se presentará con las preguntas ¿quées? ¿cómo es? La perspectiva sigue en la entidad que funciona gramatical mente como sujeto, pero no para decirnos al go en relación con el espacio que le rodea, sino para hablar de ella misma; es decir, la metáfora podría traducirse como sucesivos acercamientos (zoom) de una cámara.

6. Una cuarta unidad debe integrar todos los usos de los tres verbos.

Fig. 5.

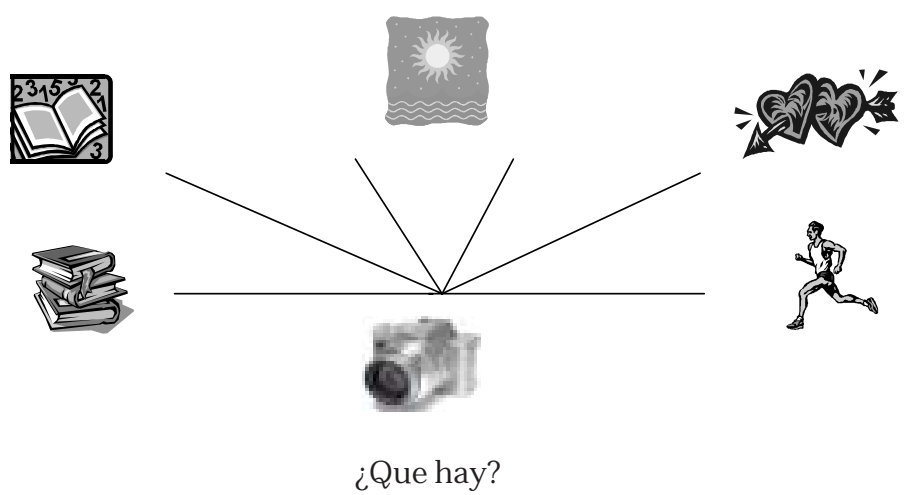


Gráficamente, la presentación de la explicación se podría hacer de esta manera:
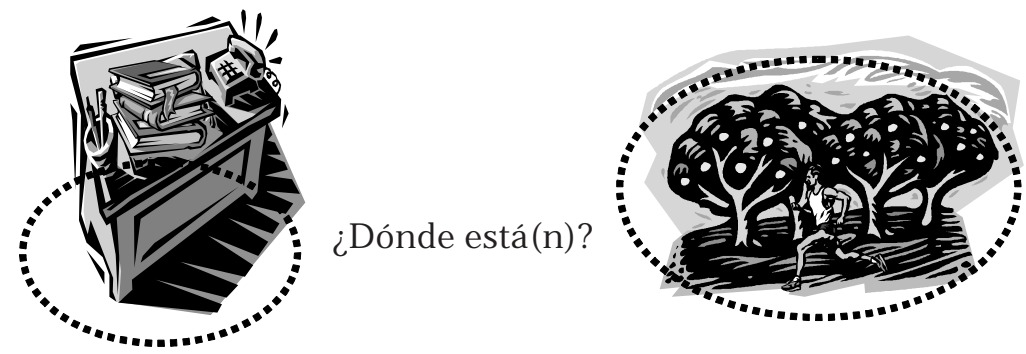

Fig. 7

\section{¿Qué es/ son? \\ ¿Cómo es/ son? \\ ¿Cuántos son?}
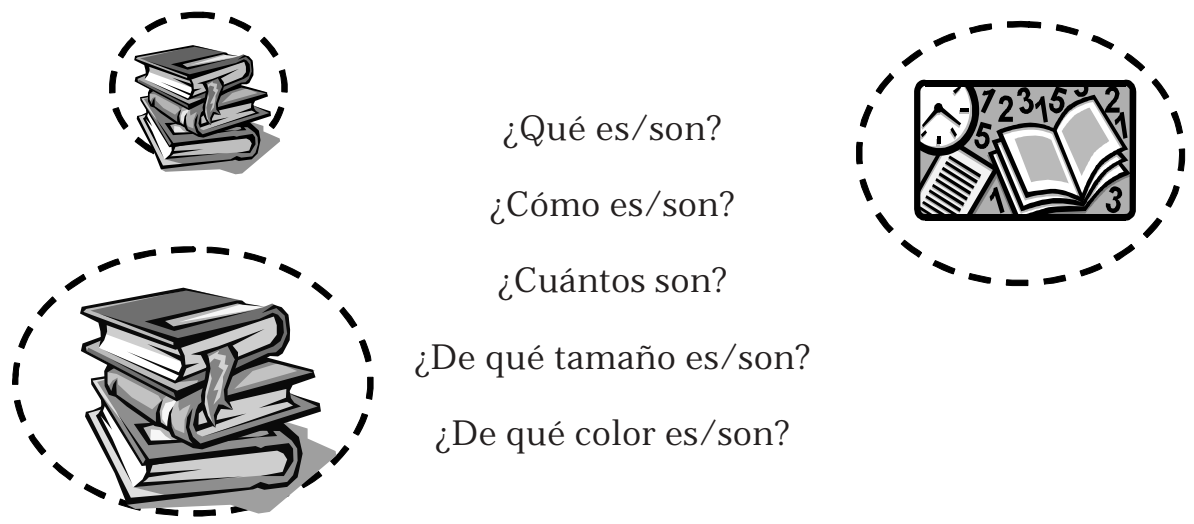

¿De qué tamaño es/ son?

¿De qué color es/ son?

Fig. 6

7. Esta cuarta unidad debe resal tar los usos conflictivos entre haber y estar y entre ser y estar; particularmente, la coincidencia de sery estar en la expresión de la relación locativa - cuando el primer argumento del predicado es un término que designa un evento (la reunión es en el salón 12) - y la combinación con adjetivos susceptibles de ser conceptualizados como producto de un cambio de estado (es tristevs. está triste). Este último uso se presentaría en relación con los verbos pronominales ( se entristeció $\rightarrow$ está triste) y presentando el esquema que aparece en la figura 4). 


\section{CONCLUSIONES}

En este artículo se han planteados tres hipótesis: Ia primera, en relación con la necesidad de recurrir a conceptos abstractos y a metáforas para comprender y explicar la complejidad de los fenómenos lingüísticos. La segunda en relación con la perspectiva impersonal izada introducida por el verbo haber. La tercera caracteriza a ser como un verbo semánticamente vacío y aspectualmenteimperfecto, mientras que estar es caracterizado como una semicópula que crea un efecto perfectivizador. Hay que aclarar que el sentido locativo atribuido tradicionalmente a estar sería una consecuencia del "efecto perfectivizador" proyectado sobre un dominio físico, y requiere, por tanto, de su argumento locativo, como queda claro en los ejempl os del cuadro uno en los que es necesario agregar al gún elemento para que la oración resulte gramatical [una casa está (ahî)].

A reserva de una mayor investigación (teórica y aplicada al aula) que permita detectar las debilidades de una propuesta de este tipo, considero que esta aproximación muestra cómo la comprensión delos fenómenos lingüísticos problemáticosatravés de conceptos semántico-cognoscitivos permite aterrizar en la simplicidad de dibujos y preguntas que guían el aprendizaje de manera control ada. Considero que explicaciones de este tipo, aunadas al desarrollo didáctico de unidades de aprendizaje, contribuirían a facilitar nuestro trabajo en el aula de español como lengua extranjera haciéndolo más fluible para el maestro y más provechoso para los estudiantes.

\section{BIBLIOGRAFÍA}

BOSQUE, I., "Sobre el aspecto en los adjetivos y en los partici pios", en Tiempo y aspecto en español. Madrid, Cátedra, 1990.

BULL, W., "Related Functions of Haber and Estar", en Modern Language J ournal, núm. 27. 1943, pp. 119-123. CUENCA, J oseph Maria y J oseph HILFERTY, Introducción a la lingüística cognitiva. Barcelona, Ariel, 1999. 
FEARN, Nicholas, Zenón y la tortuga. Barcelona, Grijal bo, 2003.

GARCÍA FERNÁNDEZ, Luis, El aspecto gramatical en la conjugación. Madrid, Arco / Libros, 1998.

HENGEVELD, Kees, "Copular verbs in a functional grammar of Spanish", en Linguistics, núm. 24. 1986, pp. 393-420.

LANGACKER, Ronald, Foundations of Cognitive Grammar: Theoretical Prerequisites. Cal ifornia, Stanford University Press, 1987.

LUQUE MORENO, J ., "En torno al sintagma «Haber impersonal + sustantivo»y sus orígenes latinos", en Revista española de lingüística. Órgano de la Sociedad Española de Lingüística, año 8. 1978, pp. 125-147.

LYONS, J ohn, "A note on possessive, existential and locative sentences", en Foundations of Language, núm. 3. 1967. pp. 390-396.

—, Semántica. Barcelona, Teide, 1977.

MolinA, Redondo, J . A. de y J. Ortega Olivares, Usos de ser y estar. Madrid, SGEL, 1996. 
